\title{
Outcomes of neonatal jaundice in Taiwan
}

\author{
Pei-Chen Tsao, ${ }^{1,2,3,4}$ Hsin-Ling Yeh, ${ }^{5}$ Yen-Chen Chang, ${ }^{5}$ Po-Huang Chiang, ${ }^{5}$ \\ Yu-Shih Shiau, ${ }^{6}$ Szu-Hui Chiang, ${ }^{6}$ Wen-Jue Soong, ${ }^{1,2,4}$ Mei-Jy Jeng, ${ }^{1,2,4}$ \\ Kwang-Jen Hsiao ${ }^{6}$
}

'Division of General Pediatrics, Pediatrics Department, Taipei Veterans General Hospital, Taipei, Taiwan

Department of Pediatrics, School of Medicine, National Yang-Ming University, Taipei, Taiwan

${ }^{3}$ Institute of Physiology, School of Medicine, National YangMing University, Taipei, Taiwan

${ }^{4}$ Institute of Emergency and Critical Care Medicine, School of Medicine, National Yang-Ming University, Taipei, Taiwan

The Institute of Population Health Sciences, National Health Research Institutes, Zhunan, Taiwan

${ }^{6}$ Preventive Medicine Foundation, Taipei, Taiwan

\section{Correspondence to}

Professor Kwang-Jen Hsiao, Preventive Medicine Foundation, Taipei 10699, Taiwan; hsiao@pmf.tw

Received 5 September 2017 Revised 30 January 2018 Accepted 5 February 2018 Published Online First 22 February 2018
Check for updates

(C) Author(s) (or their employer(s)) 2018. No commercial re-use. See rights and permissions. Published by BMJ.

To cite: Tsao P-C, Yeh $\mathrm{H}-\mathrm{L}$, Chang Y-C, et al.

Arch Dis Child

2018:103:927-929.

\section{ABSTRACT}

Objective To investigate the burden of clinically significant neonatal jaundice (SNJ) in Taiwan, 20002010.

Study design The nationwide, population-based health insurance database in Taiwan was used to investigate the incidence, kernicterus rate and mortality rates of SNJ cohort born between 2000 and 2010.

Results From 2000 to 2010, up to 242546 patients admitted with neonatal jaundice (NJ) were identified. The incidence of SNJ was $5.9 \%$ in 2000 and increased to $13.7 \%$ in $2010(P<0.001)$. The mortality rate significantly decreased from $0.51 \%$ in 2000 to $0.26 \%$ in $2010(P<0.001)$ and the average incidence of kernicterus was 0.86 per 100000 live births, indicating dramatically decreased rates compared with earlier rates in Taiwan. Conclusions In spite of the increased incidence rates, the rates of mortality and kernicterus in patients with NJ significantly declined in Taiwan. The public health prevention programme, clinicians' awareness and effective management might contribute to the reduction of these acute severe sequelae.

\section{INTRODUCTION}

Neonatal jaundice (NJ) can be a benign physiological process, or it can also be the first sign of serious illness with bilirubin-associated toxicity manifested in the nervous system. The practice guidelines released by the American Academy of Pediatrics (AAP) have been well accepted to monitor and identify newborns who might have severe unconjugated hyperbilirubinaemia, and even acute bilirubin encephalopathy or kernicterus. ${ }^{1}$ Severe neonatal hyperbilirubinaemia still contributes significantly to neonatal morbidity and mortality, especially in low/ middle-income countries. ${ }^{2}$

A hospital-based report showed very high rates of mortality and kernicterus of all neonates admitted due to NJ during the 1970 s in Taiwan. ${ }^{3}$ There was a lack of data using relatively large populations to investigate the current situation and outcomes of NJ in Taiwan. In this study, we used a nationwide population-based data set from 2000 to 2010 to survey the burden of neonatal hyperbilirubinaemia in Taiwan.

\section{METHODS}

This retrospective, population-based study was conducted using data from the Taiwan National Health Insurance Research Database (NHIRD) released by the Taiwan National Health Research Institutes (NHRI) (http://nhird.nhri.org.tw/en/). Personal identification is encrypted before the

\section{What is already known?}

- During the 1970s, of all neonates admitted due to neonatal jaundice (NJ), $16 \%$ died and 32\% were diagnosed with kernicterus in Taiwan.

- NJ is still a burden of public health, especially in low/middle-income countries.

- Severe neonatal hyperbilirubinaemia causes adverse acute and long-term neurological consequences.

\section{What this study adds?}

- NJ remains a common disease at the neonatal stage during the first decade of the 21 st century in Taiwan.

- With the efforts of public health prevention programmes and clinical managements, the mortality, exchange transfusion and kernicterus rates for NJ decreased dramatically in Taiwan.

release of the data set for public access. The database includes comprehensive information on insured subjects, such as demographic data, dates of clinic visits, disease diagnosis and interventions. The diagnostic codes of the patients in the NHIRD are established by board-certified physicians in their corresponding specialties with the format of the International Classification of Diseases, Ninth Revision, Clinical Modification (ICD-9-CM).

Significant neonatal jaundice (SNJ) cases were identified from a Specific Subject Dataset (SSD) which comprised all records of subjects who were hospitalised with NJ diagnostic code (ICD-9-CM, 774) in the NHIRD from 2000 to 2010. The process of SNJ case extraction is shown in figure 1. Newborn subjects diagnosed with NJ within 31 days of age were selected as SNJ cohort 2000-2010. For newborns without their own records at neonatal stage, records with a birth code (ICD-9-CM, V3) and/or perinatology code (ICD-9-CM, 76 and 77) were distinguished from the mothers' records and reconfirmed the coding accuracy. Using the procedure codes of phototherapy (ICD-9-CM, 99.83) and exchange transfusion (ET; ICD-9-CM, 99.01), the data on interventions for SNJ were also collected. According to the treatment codes in the NHIRD, the intervention of phototherapy was classified as simple and intensive phototherapy. The National Health Insurance Administration (NHIA) had set up the criteria of different interventions for 


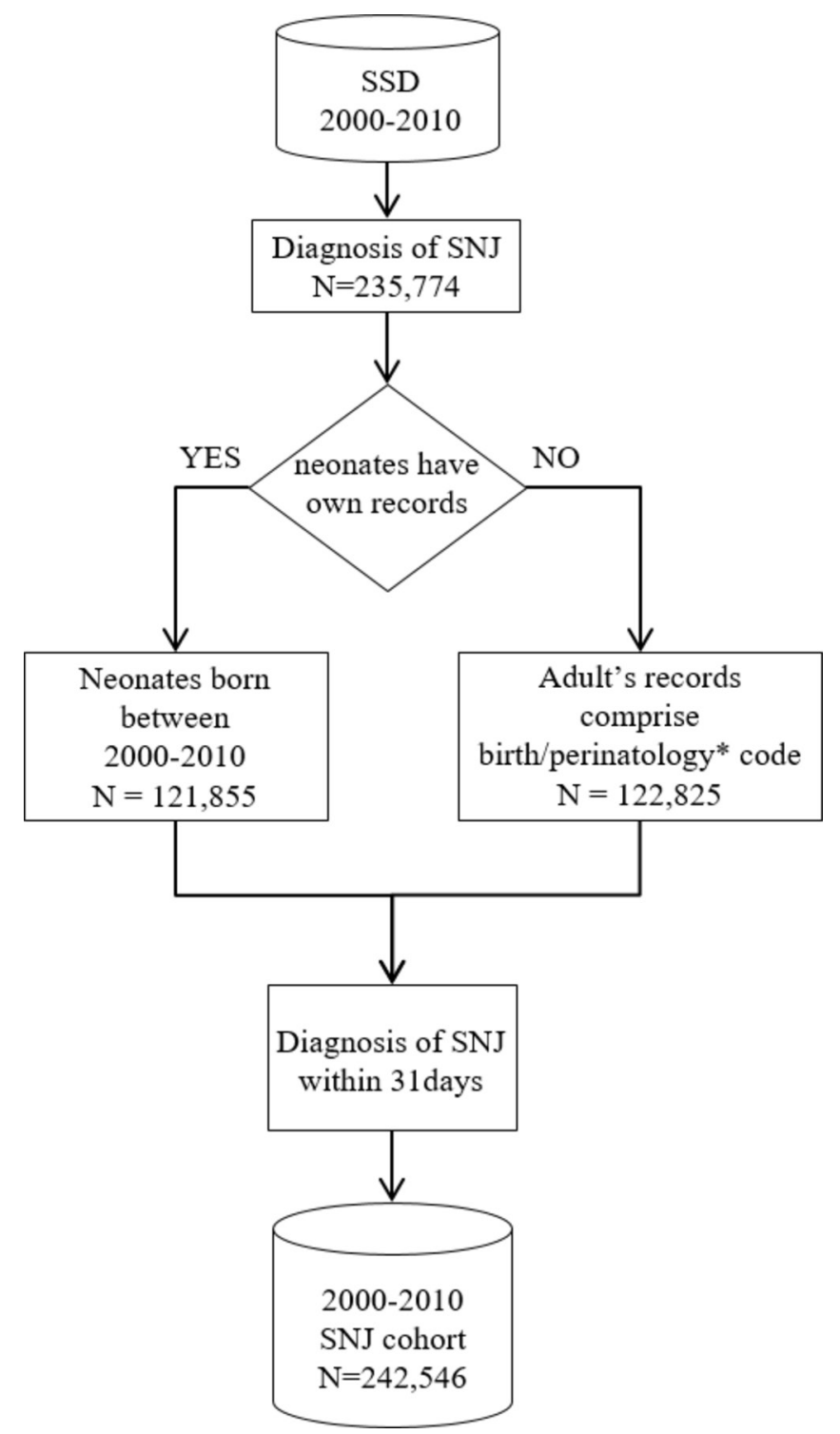

Figure 1 Flow chart of study subjects extracted from Specific Subject Dataset (SSD). SSD is composed of all medical records of subjects hospitalised with neonatal jaundice (NJ) diagnostic code (ICD-9-CM, 774) in the National Health Insurance Research Database (NHIRD) between 2000 and 2010. *Birth code: ICD-9-CM, V3. Perinatology code: ICD-9-CM, 76 and 77. N, subject number; SNJ, significant neonatal jaundice.

$\mathrm{NJ}$ according to the AAP recommendations. Based on the guidelines for phototherapy and ET by AAP, physicians took patient's age, risk factors and bilirubin level into account and decided the medical interventions. NHIA audited these interventions of sampled claims routinely. In this study, we categorised these patients by three levels of intervention, phototherapy, intensive phototherapy and ET, which were supposed corresponding to moderate, severe and extreme NJ.

\section{Statistical analysis}

The incidences and mortality rates of each year were measured and tested for trend in proportions. Two-tailed $\mathrm{P}$ values of less than 0.05 were considered statistically significant for all analyses. All data management and analyses were performed using a free program R (http://cran.r-project.org/).

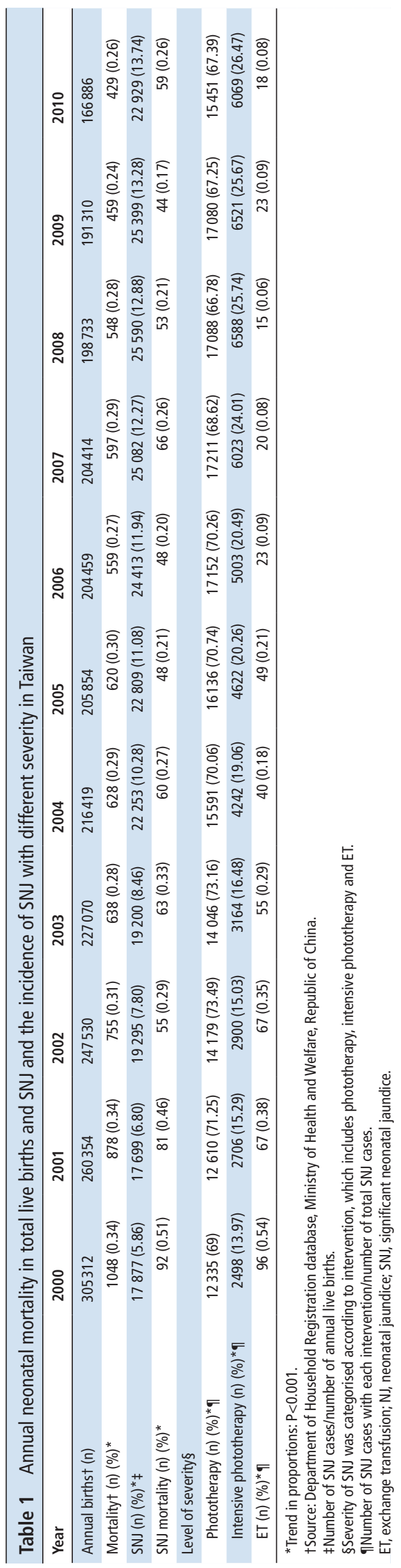




\section{RESULTS}

During the study period, 2000-2010, a total of 242546 neonates admitted and diagnosed with $\mathrm{NJ}$ were selected from the SSD 2000-2010 of NHIRD as SNJ cohort 2000-2010 (figure 1). Most of the enrolled newborns (98.4\%) had been admitted with diagnosis of NJ within 14 days old. The annual mortality of live births and SNJ, incidence of SNJ and severity categorised in accordance with the managements are shown in table 1. As shown, the number of yearly live births in Taiwan declined steadily. On the contrary, the case numbers and incidence of SNJ over the same period increased significantly (from 17877 (5.9\%) in 2000 to $22929(13.7 \%)$ in 2010; $\mathrm{P}<0.001)$. The number of cases requiring intensive phototherapy increased significantly (from 2498 (14\%) to 6069 (26.5\%), respectively; $\mathrm{P}<0.001$ ), and those requiring ET decreased significantly (from 96 (0.54\%) to $18(0.08 \%)$, respectively; $\mathrm{P}<0.001)$. The incidence of ET remained steadily low in SNJ cohort 2000-2010 over the last 5 years. There was a significant decrease in mortality rate from $0.51 \%$ in 2000 to $0.26 \%$ in $2010(\mathrm{P}<0.001)$. This cohort had a significantly higher mortality rate than did the total live births in 2000 and 2001, and declined afterwards to close to or even less than did the total live births. Kernicterus was diagnosed within 1 month of age in one to three patients per year in this cohort.

\section{DISCUSSION}

This nationwide population-based study was done to review the incidence, kernicterus and mortality rates of SNJ cohort 20002010 in Taiwan. Our results showed that the incidence rates of SNJ more than doubled during the study period. Although the clinical practice guideline for diagnosis of $\mathrm{NJ}$ has updated in $2004,{ }^{1}$ which might contribute to the increase of NJ detection rate, the incidence of $\mathrm{NJ}$ in Taiwan has a steady increasing trend during the entire study period, 2000-2010, both before and after the guideline modification. According to the report of Taiwan Birth Registry, the number of live births with $<37$ weeks of gestation had slightly increased from $8.92 \%$ in 2004 to $9.13 \%$ in 2010. In addition to these factors, the most probable factor associated with the increased incidence rate was an increase in the breast feeding rates at 1 month after delivery in Taiwan (from $35.9 \%$ in 1996 to $54.2 \%$ in 2004 to $86.9 \%$ in 2010 ), especially in the rates of exclusive breast feeding (from 5\% to $46.6 \%$ to $65.7 \%$, respectively) (http://www.pitt.edu/ super7/4901150001/49111-49121.pdf). The mortality rates and the number of patients in SNJ cohort 2000-2010 who needed ET declined significantly. Kernicterus occurred in one to three cases per year, indicating dramatically decreased numbers compared with earlier data in Taiwan. ${ }^{3}$

Previous studies reported varied worldwide incidence rates of severe hyperbilirubinaemia about $0.02-0.12 \%$ live births ${ }^{4}$ in high-income countries, and 68-491\%o in mid-income or low-income countries. ${ }^{5}$ High rates of kernicterus $(16.8 \%-31.6 \%)$ and mortality (9.2\%-15.8\%) were found in NJ cases admitted in Taiwan during the 1970 s. $^{3}$ In our study, the nationwide incidence of patients in SNJ cohort 2000-2010 requiring phototherapy increased significantly. However, the incidence of these patients requiring ET decreased significantly, as did the mortality rates. The average incidence of kernicterus was 0.86/100 000 live births, which was compatible with estimated incidence in developed countries. ${ }^{2}$ A universal newborn screening programme for G6PD deficiency launched in Taiwan since 1987 was dedicated to avoid the occurrence of haemolytic NJ. ${ }^{6}$ Physicians and healthcare providers routinely provided education resources for parents and screened all neonates based on the cephalocaudal progression scale before discharge and in the following clinics. In addition, other risk factors associated with jaundice, such as preterm, were also taken into consideration. When hyperbilirubinaemia was suspected after discharge from birth facilities, these neonates would be referred to clinics by nursing staff in postpartum care facilities or be brought to clinics by their family alone from home. According to physicians and healthcare providers' judgements, transcutaneous bilirubin screening would be offered for those infants in risk of clinically significant jaundice. In addition to the increased breast feeding rates and other factors, the clinical awareness might result in the increased rates of hospital admission due to NJ (SNJ), but early and aggressive management possibly contributes to the reduction in rates of severe hyperbilirubinaemia and patients requiring ET in Taiwan.

In conclusion, NJ remains a common disease at the neonatal stage but the rates of mortality and kernicterus were dramatically decreased in Taiwan. A variety of efforts, including the public health prevention programme (including universal newborn screening for G6PD deficiency), clinicians' awareness and effective management, might contribute to the reduction in these acute severe sequelae caused by NJ during the first decade of the 21st century in Taiwan.

Contributors $\mathrm{KJH}$ conceptualised and designed the study, reviewed and revised the manuscript, and approved the final manuscript as submitted. PHC conceptualised and designed the study, data collection and analysis, and approved the final manuscript as submitted. PCT carried out the initial analyses, drafted the initial manuscript and approved the final manuscript as submitted. HLY and YCC performed the database operation, data analysis, and approved the final manuscript as submitted. YSS and SHC coordinated, supervised data collection and analysis, and approved the final manuscript as submitted. WJS and MJJ supervised the initial manuscript, and approved the final manuscript as submitted.

Funding This research received no specific grant from any funding agency in the public, commercial or not-for-profit sectors.

Competing interests None declared.

Ethics approval This study was approved by the NHRI and the Institutional Review Board of the NHRI (IRB approval number: EC1041107-E)

Provenance and peer review Not commissioned; externally peer reviewed.

\section{REFERENCES}

1 American Academy of Pediatrics Subcommittee on Hyperbilirubinemia. Management of hyperbilirubinemia in the newborn infant 35 or more weeks of gestation. Pediatrics 2004;114:297-316.

2 Kaplan M, Bromiker R, Hammerman C. Severe neonatal hyperbilirubinemia and kernicterus: are these still problems in the third millennium? Neonatology 2011;100:354-62.

3 Chang MH, Chen SH, Lee TC. Neonatal Hyperbilirubinemia and Erythrocyte G-6-PD Deficiency in Chinese Newborn Infants. Acta Paediatr Sin 1977;18:1-10.

4 Zoubir S, Mieth RA, Berrut S, et al. Incidence of severe hyperbilirubinaemia in Switzerland: a nationwide population-based prospective study. Arch Dis Child Fetal Neonatal Ed 2011;96:F310-F311.

5 Greco C, Arnolda G, Boo NY, et al. Neonatal Jaundice in Low- and Middle-Income Countries: Lessons and Future Directions from the 2015 Don Ostrow Trieste Yellow Retreat. Neonatology 2016;110:172-80

6 Chiang SH, Wu SJ, Wu KF, et al. Neonatal screening for glucose-6-phosphate dehydrogenase deficiency in Taiwan. Southeast Asian J Trop Med Public Health 1999;30(Suppl 2):72-4. 